\title{
O Prêmio Nobel de Química de 2010, Acoplamentos Cruzados e o BMOS - 14
}

O 14th Brazilian Meeting on Organic Synthesis (BMOS-14), realizado em Brasília em setembro de 2011, trouxe ao Brasil o professor Akira Suzuki, laureado, juntamente com os professores Ei-ichi Negishi e Richard Heck, com o prêmio Nobel de Química de 2010. Esses pesquisadores receberam o Prêmio Nobel por seus estudos nas aplicações do metal paládio em processos químicos de grande utilidade para a sociedade - produção de fármacos e novos materiais. Os professores Suzuki e Negishi fizeram extensos estudos em reações conhecidas como acoplamentos cruzados. Neste manuscrito, traçamos um breve histórico do desenvolvimento dessas reações de acoplamento cruzado, incluindo os acoplamentos de Negishi e Suzuki.

Palavras chave: Prêmio Nobel, acoplamentos cruzados, paládio.

The 14th Brazilian Meeting on Organic Synthesis (BMOS-14), held in Brasilia on September 2011, brought Professor Akira Suzuki to Brazil as a plenary speaker at the event. Professor Suzuki, together with Professors Ei-ichi Negishi and Richard Heck were the Nobel Prize laureates in 2010. These researchers received the Nobel Prize for their work on the use of palladium metal in organic transformations of enormous utility to society - production of medicines and new materials. Professors Suzuki and Negishi performed most of their studies on the development of reactions known as crosscouplings. This manuscript presents the development of the cross-coupling reactions, including the Negishi and Suzuki coupling reactions.

Keywords: Nobel Prize, cross-coupling reactions, palladium. 


\section{Introdução}

O $14^{\circ}$ Brazilian Meeting on Organic Synthesis (BMOS-14), realizado em Brasília entre os dias 1 e 5 de setembro de 2011, foi um evento científico especial para a comunidade química brasileira. O fato que tornou o BMOS-14 tão especial foi a presença do professor Akira Suzuki, laureado com o Prêmio Nobel de Química de 2010, como palestrante no evento. Desta forma, os BMOS refletem o contínuo amadurecimento da comunidade química brasileira, em sintonia com os acontecimentos científicos mais relevantes da área em termos mundiais.

O professor Akira Suzuki, juntamente com os professores Ei-ichi Negishi e Richard Heck, compõe o grupo dos três pesquisadores laureados com o Prêmio Nobel de Química de 2010 por suas pesquisas no uso de paládio na formação de ligações carbono-carbono ${ }^{1,2}$. A premiação do Nobel de 2010 destaca esses processos catalíticos como de extrema importância na construção de moléculas orgânicas para uso em medicina e na indústria em geral.

O professor Akira Suzuki, em particular, desenvolveu um novo processo de acoplamento entre haletos de arila e/ou vinila e ácidos borônicos, conhecido como acoplamento cruzado de Suzuki, ou simplesmente acoplamento de Suzuki. Este processo foi desenvolvido em associação com o hoje professor Norio Miyaura, na Universidade de Hokkaido, no Japão, em 1979³, seguindo desenvolvimentos na área dos acoplamentos cruzados, que tiveram início em 1972 com os estudos do professor Kumada, na Universidade de Kioto, Japão, e Corriu, na França ${ }^{4}$.

Na década de 1970, o emprego de paládio firmouse em diversos acoplamentos cruzados, tornando tais acoplamentos uma ferramenta química poderosa e demonstrando ser este um processo catalítico de alto potencial científico e tecnológico ${ }^{4}$.

Uma das razões para o destaque alcançado por essas reações ao longo das últimas décadas foi a sua grande capacidade de aplicação em processos industriais, com destaque aqui para a indústria farmacêutica, que produz hoje diversos fármacos de forma econômica e em grandes quantidades, empregando essas reações com segurança e de forma ambientalmente amigável em razão do baixo volume de resíduos produzidos. Além de fármacos, esses acoplamentos cruzados têm sido aplicados em grande escala na geração de compostos importantes para a indústria eletrônica.

Embora o uso dos acoplamentos cruzados empregando paládio seja hoje quase uma rotina em laboratórios acadêmicos ou industriais, figurando ao lado das "grandes reações”, tais como a reação de DielsAlder e as olefinações de Wittig, a história por trás do desenvolvimento desses processos não aconteceu em um momento único e, ao contrário de outras descobertas, envolveu um número significativo de pesquisadores, que a partir do contato com essas reações de acoplamento acabaram escrevendo seus nomes na história da Química.

\section{O ACOPLAMENTO DE KUMADA-TAMAO- CORRIU (1972): OS “PRIMEIROS” ACOPLAMENTOS CRUZADOS}

O acoplamento de haletos de alquenila ou arila com reagentes de Grignard promovidos por catalisadores de Ni constituem os primeiros exemplos de acoplamentos cruzados relatados na literatura e são creditados aos trabalhos iniciais de Kumada ${ }^{5}$. Curiosamente, e de forma quase simultânea, Robert Jean Pierre Corriu, trabalhando de forma independente em Montpellier, França, relatou uma forma de acoplamento praticamente idêntico ${ }^{6}$. Por isso, este acoplamento cruzado empregando sais de níquel e reagentes de Grignard é hoje conhecido como o acoplamento de Kumada-Tamao-Corriu. O nome de Corriu é frequentemente omitido nas referências a esse acoplamento, provavelmente porque o grupo de Kumada no Japão se mostrava mais ativo em uma linha metodológica-sintética do que o grupo de Corriu, na França, e também porque a escola formada por Kumada, em Kioto, teve seguidores fieis e extremamente dedicados. Mesmo o nome de Tamao é frequentemente omitido e o acoplamento é simplesmente relatado hoje em dia como acoplamento de Kumada.

A racionalização para o acoplamento formulada por Kumada e Tamao tinha o formato a seguir, válido até hoje em seus princípios básicos (Esquema 1).

É importante salientar que Kumada, assim como Corriu, tinham conhecimento dos trabalhos anteriores de A. Yamamoto, que havia relatado, em 1970, seus resultados sobre a reatividade de um complexo 
dietilníquel, levando a produtos de homoacoplamento em bons rendimentos (Equação 1) ${ }^{7}$. Yamamoto é o autor de expressões ainda hoje utilizadas ao descrever as diversas etapas do processo de acoplamento, tais como adição oxidativa, transmetalação e eliminação redutiva. Essas mesmas etapas foram reconhecidas por Kumada e Tamao em seus estudos envolvendo haletos de arila e os reagentes de Grignard. A razão pela qual Yamamoto não aparece como descobridor do processo de acoplamento cruzado é porque ele não reconheceu o potencial sintético desta transformação, estando mais interessado nas propriedades do complexo de níquel e no mecanismo da transformação. Essa "distração" é um aspecto do qual ele se arrepende amargamente até os dias de hoje ${ }^{8}$.

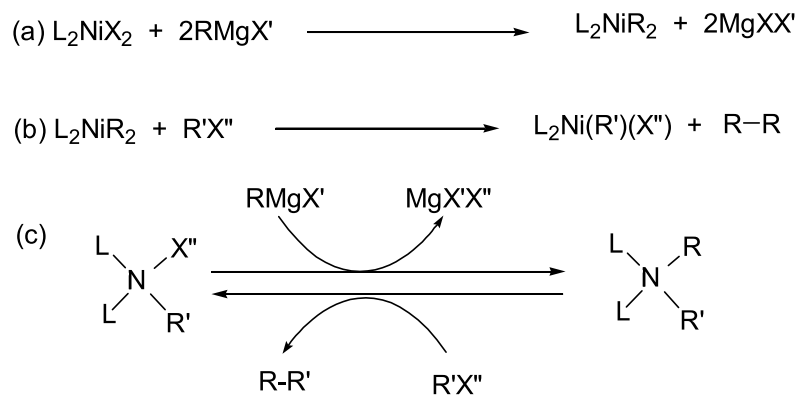

Esquema 1. O acoplamento de Kumada-Tamao-Corriu, como proposto em $1972^{5}$

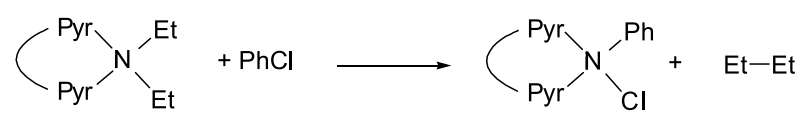

Equação 1. Os resultados de Yamamoto nos estudos de homoacoplamento ${ }^{7}$

Para efeitos de precisão nas informações, é preciso lembrar ainda os primeiros resultados envolvendo homoacoplamentos promovidos por metais de transição a partir de reagentes de Grignard, efetuados por Morris Karasch e Jay Kochi ${ }^{9}$. No entanto, esses pesquisadores estavam mais interessados na química dos radicais de carbono e no mecanismo desses processos e não vislumbraram os homoacoplamentos como um processo de valor sintético.

Alguns resultados dos trabalhos iniciais de KumadaTamao e de Corriu são apresentados nos Esquemas 2 e 3 a seguir.

\section{O ACOPLAMENTO CRUZADO DE MURAHASHI (1975)}

O emprego de paládio, hoje tão disseminado nos acoplamentos cruzados, teve origem com os estudos de Murahashi. Os estudos com Pd foram iniciados porque os catalisadores utilizados até aquele momento (1975) não eram eficientes na realização de acoplamentos cruzados envolvendo os reagentes organolítio - de uso mais geral e mais facilmente obtidos do que os reagentes de Grignard. Como Murahashi já havia conseguido realizar a alquilação de acetatos de alila com RLi empregando $\mathrm{Pd}$, ele resolveu testar os reagentes organolitiados no acoplamento cruzado com haletos de alquenila, na presença de $\operatorname{Pd}(0)^{10}$. Portanto, Murahashi foi o primeiro a utilizar Pd na realização dos acoplamentos cruzados (Esquema 4).

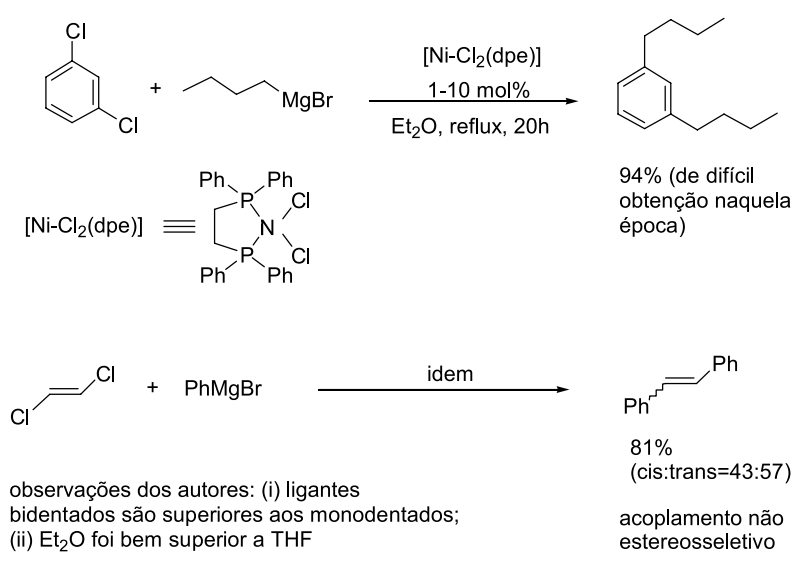

Esquema 2. Alguns dos resultados iniciais de Kumada-Tamao ${ }^{5}$

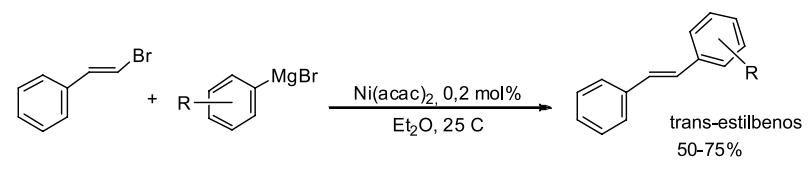

$\mathrm{R}=$ 4-MeO, 4-Me, 3-Me, 4-Br, 2,4-diMe, alfa-naftila, 2-tienila

outros catalisadores testados: $\mathrm{NiCl}_{2},\left(\mathrm{PPh}_{3}\right)_{2} \mathrm{NiCl}_{2}$ e CoCl${ }_{2}$ melhores resultados foram obtidos com $\mathrm{Ni}(\mathrm{acac})_{2}$

Esquema 3. Alguns dos resultados de Corriu ${ }^{6}$

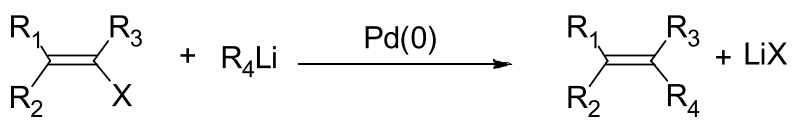

Esquema 4. A reação geral do acoplamento de Murahashi empregando $\mathrm{Pd}^{10}$ 
Alguns exemplos dos trabalhos de Murahashi são apresentados a seguir (Esquemas 5 e 6$)^{11}$.

\section{O ACOPLAMENTO DE NEGISHI (1976)}

Ei-ichi Negishi teve participação decisiva no desenvolvimento dos acoplamentos cruzados envolvendo haletos de arila e alquenila com derivados organoalumínio, organozinco, organozircônio, organoboro e organoestananas ${ }^{12}$.

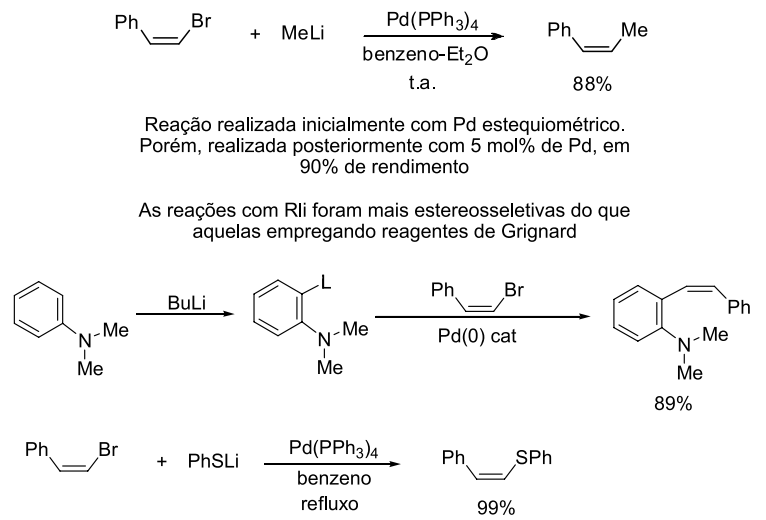

Esquema 5. Algumas das primeiras reações de acoplamento cruzado utilizando $\mathrm{Pd}^{11}$

O ímpeto inicial dos trabalhos foi adaptar o acoplamento de Kumada (Ni como catalisador) a outros organometálicos que não os reagentes de Grignard. Os primeiros experimentos de Negishi envolveram o acoplamento de haletos de arila com alquenilalanos, catalisado por $\mathrm{Ni}(0)$. O uso de alquenilalanos foi consequência direta dos estudos de Negishi com a reação de hidroaluminação de alcinos (Esquema 6).

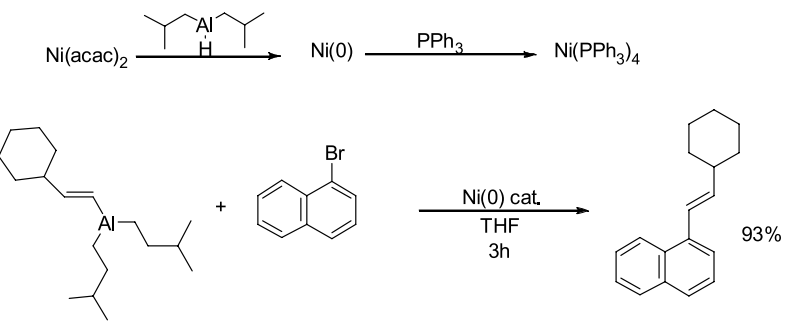

Esquema 6. Os resultados iniciais de Negishi envolvendo alquenilalanos $^{12}$

Curiosamente, nesses primeiros resultados, Negishi menciona que o uso de $\mathrm{Pd}$ não trazia vantagens sobre o uso de $\mathrm{Ni}(0)$, e que o uso de aluminatos em substituição aos alanos acarretava drástica redução no rendimento do acoplamento (de 93\% para 19\%). Ele relatou também que o uso de organoboranos e de seus respectivos complexos "ato" $\left(\mathrm{R}_{4} \mathrm{BLi}\right)$ não conduzia a produtos de acoplamento. A grande ironia aqui é que um ano mais tarde o uso de reagentes de boro neste tipo de acoplamento daria origem ao acoplamento de SuzukiMiyaura, um dos mais importantes acoplamentos cruzados e largamente utilizado em sínteses orgânicas, inclusive em escala industrial.

Em trabalhos posteriores de Negishi, já se nota uma maior tendência ao uso de $\operatorname{Pd}(0)$ nos acoplamentos, havendo inclusive uma grande superioridade sintética em favor do Pd. Como demonstra o exemplo do Esquema 7, as reações utilizando Pd forneceram maiores rendimentos e se mostram mais estereosseletivas do que aquelas empregando Ni como catalisador ${ }^{2}$.

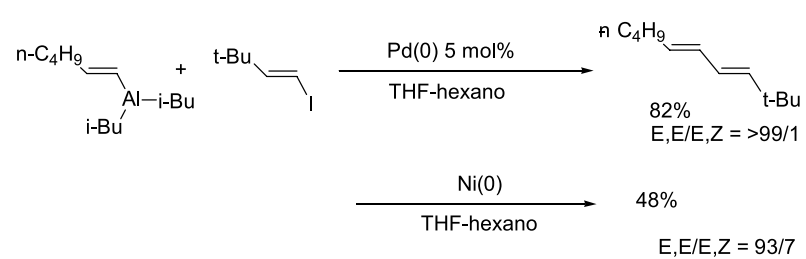

Esquema 7. Aplicações do acoplamento de Negishi ${ }^{2}$

Negishi é também reconhecido como pioneiro no uso de compostos organozinco e organozircônio em acoplamentos cruzados. Via de regra, esses acoplamentos são bastante eficientes, podendo ocorrer à temperatura ambiente ou mesmo em temperaturas mais baixas (Esquema 8).

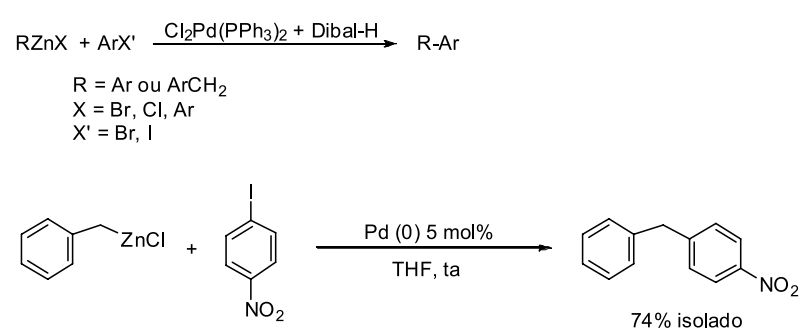

Esquema 8. Acoplamentos de Negishi envolvendo compostos organozinco $^{2}$

O acoplamento empregando derivados organozircônio apresenta algumas vantagens do ponto 
de vista da formação do organoderivado, pois a reação de hidrozirconação é compativel com diversos grupos funcionais - COOMe, CN, OMe, $\mathrm{Cl}$ e outras funções oxi (OR). Os derivados organozircônio ainda gozam da vantagem de poderem ser intermediários na conversão destes para os correspondentes organo-alumínio (reação do organozircônio com $\mathrm{AlMe}_{3}$ ). As reações de hidrozirconação são facilmente realizadas com o reagente de Schwartz (Cl(H)ZrCp ${ }_{2}$ ) (Esquema 9).

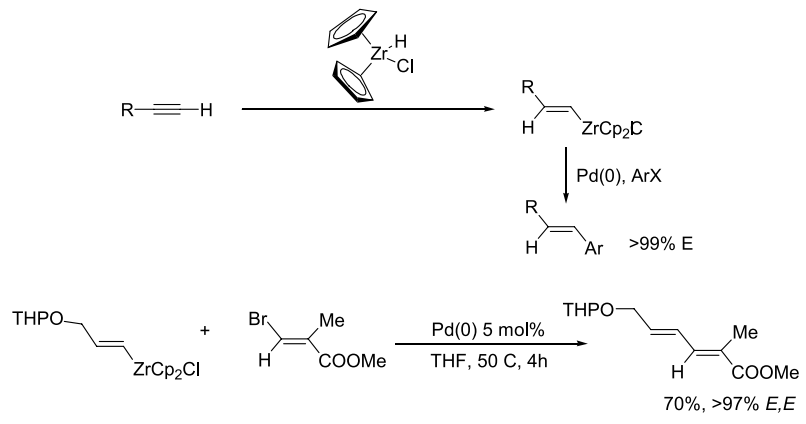

Esquema 9. Acoplamentos de Negishi empregando compostos organozircônio ${ }^{2}$

\section{ACOPLAMENTO DE SONOGASHIRA (1975)}

Esteéum acoplamento queenvolve fundamentalmente a formação de ligações entre $\mathrm{C} \mathrm{sp}{ }^{2}$ com $\mathrm{C}$ sp, permitindo conectar arilas e/ou vinilas a alcinos terminais (Esquema $10)^{13}$.

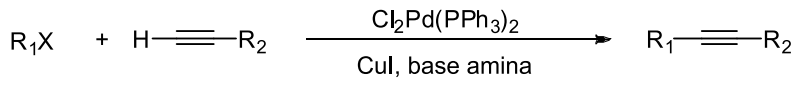

$$
\begin{aligned}
& \mathrm{R}_{1}=\text { arila ou alquenila } \\
& \mathrm{X}=\mathrm{Cl}, \mathrm{Br}, \mathrm{I} \text {, OTf } \\
& \text { base }=\mathrm{NHEt}_{2}, \mathrm{Et}_{3} \mathrm{~N} \text { ou piperidina }
\end{aligned}
$$

Esquema 10. $\mathrm{O}$ acoplamento de Sonogashira em seus aspectos gerais ${ }^{13}$

Apesar desse acoplamento levar o nome de acoplamento de Sonogashira, ele próprio é o primeiro a admitir que a reação foi também descoberta independentemente por dois outros grupos de pesquisas no ano de $1975^{14}$. Alguns pesquisadores atribuem ao prof. R. Heck a descoberta do acoplamento entre $\mathrm{C} \mathrm{sp}^{2}$ e C sp, embora os estudos mais extensos sobre esse acoplamento tenham sido realizados por Sonogashira e seu grupo. Os estudos dos outros dois grupos (Cassar e Heck) estavam mais relacionados a uma extensão da reação de Heck (formalmente não relacionada aos acoplamentos cruzados). O uso de $\mathrm{Cu}(\mathrm{I})$ foi ação exclusiva do grupo de Sonogashira e se apoiou em resultados anteriores da reação de Stephens-Castro (Esquema 11) ${ }^{15}$.

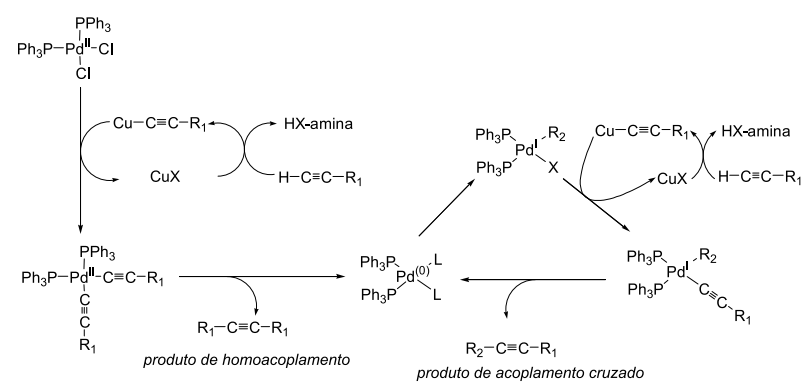

Esquema 11. O ciclo catalítico do acoplamento de Sonogashira ${ }^{15}$

\section{O ACOPLAMENTO DE STILLE}

Segundo relatos recentes, a descoberta do acoplamento cruzado envolvendo haletos de arila e alquenila com organoestananas parece ter se iniciado com Masanori Kosugi ${ }^{16}$, quando ele estudava reações de desalogenação utilizando hidretos de estanho (Esquema 12). Devido aos aspectos anti-econômicos e ambientais desse processo (hidretos de estanho são caros, tóxicos e de difícil manipulação), eles decidiram utilizar estananas orgânicas que possibilitassem a transferência de outros grupos que não o hidrogênio. Dai surgiu a transformação promovida por Indio (In), representada no Esquema $12^{16}$.

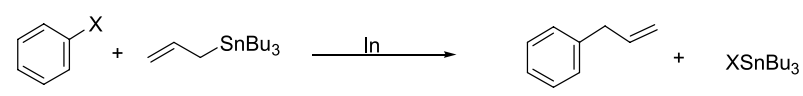

Esquema 12. O acoplamento entre haletos de arila e alilestanana, como descrito por Kosugi, em $1973^{16}$

Segundo comenta Kosugi $^{17}$, neste mesmo ano surgiu na literatura - mesmo ano e mesma revista - uma publicação de Pereyre relatando uma transformação bastante $\operatorname{similar}^{18}$. Com a divulgação dos resultados de Karasch naquele período, o grupo de Kosugi resolveu fazer a adição de outros sais de metais de transição à reação, obtendo os acoplamentos apresentados no Esquema $13^{19}$.

Essas transformações se constituem no início do “acoplamento de Stille”, embora a primeira comunicação de Stille sobre o acoplamento envolvendo compostos 
de estanho com espécies eletrofílicas de carbono (ArX e C=C-X), catalisada por Pd, tenha sido relatada somente em $1978^{20}$. O nome "acoplamento de Stille" advém da intensa participação de Stille e seu grupo nos aspectos sintéticos e mecanísticos do processo, embora a descoberta não tenha sido dele ${ }^{21}$. Alguns exemplos desse acoplamento estão ilustrados no Esquema 14.

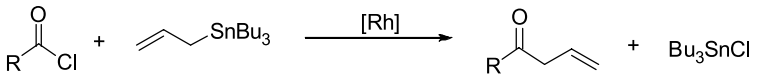

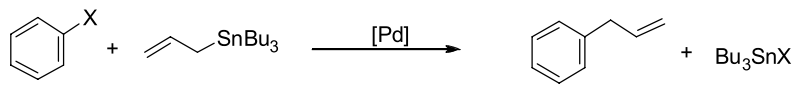

Esquema 13. Os primeiros exemplos do "acoplamento de Stille"19

As condições reacionais inicialmente relatadas por Kosugi e Stille eram relativamente drásticas (temperaturas frequentemente acima dos $100{ }^{\circ} \mathrm{C}$ ). Novos ligantes $\left(\mathrm{AsPh}_{3}\right)$ e o uso de sais de $\mathrm{Cu}(\mathrm{I})$ tornaram a reação de acoplamento bem mais branda, resolvendo em boa medida o problema de reatividade das organoestananas. Esses novos ligantes e o $\mathrm{Cu}$ aceleram a reação de transmetalação, agora feita por intermediários organocobre.

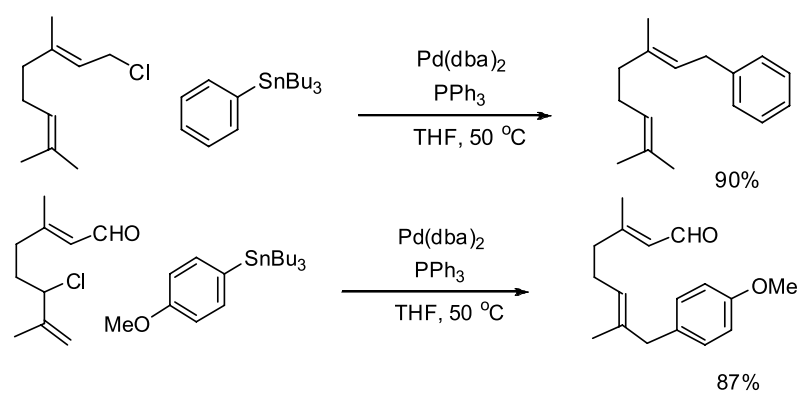

Esquema 14. Exemplos da reação de "acoplamento de Stille”21f

O mecanismo da reação de Stille-Kosugi-Migita, com incorporações mais recentes de Espinet, é apresentado a seguir (Esquema 15) ${ }^{22}$.

A despeito da tendência na redução do uso de organoestananas em síntese orgânica, o acoplamento de Stille sempre gozou de boa popularidade em muitas sínteses totais - em particular na literatura mais antiga por ser um acoplamento bastante eficiente. Essa reação foi fundamental na síntese total da (-)-macrolactina A, por Smith e colaboradores (Esquema 16) ${ }^{23}$.

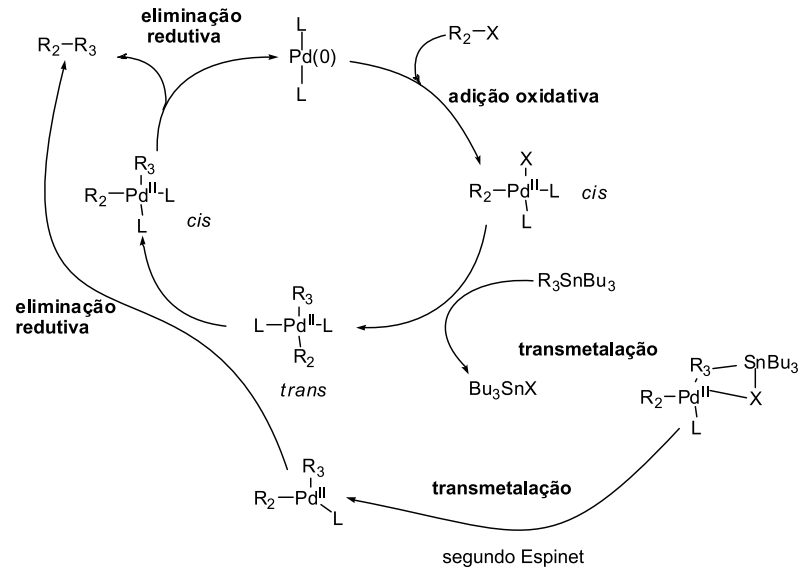

Esquema 15. O ciclo catalítico envolvido no "acoplamento de Stille”22

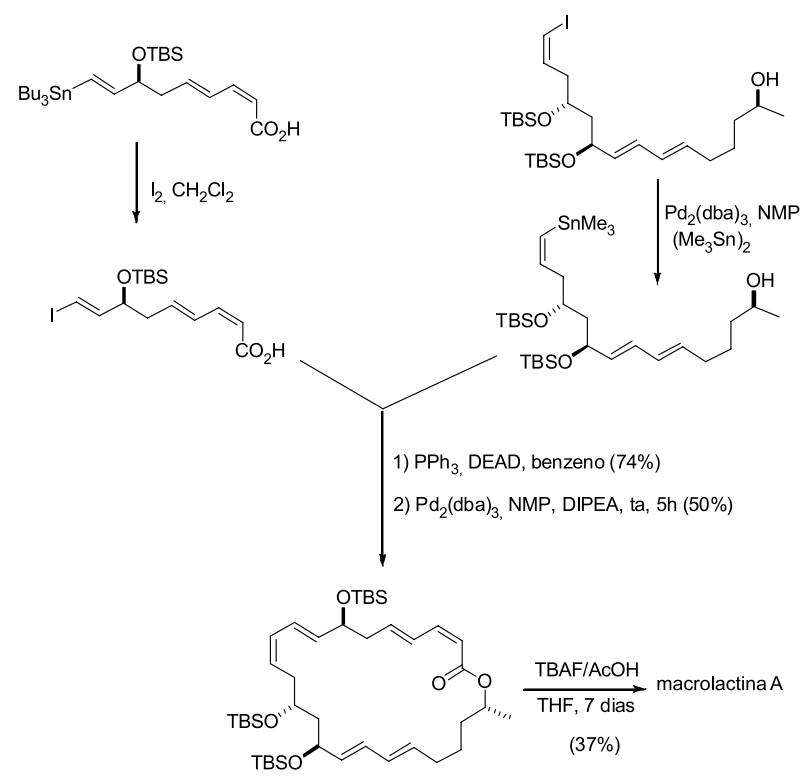

Esquema 16. Aplicação da reação de acoplamento de Stille na síntese total da macrolactina A por Smith e colaboradores ${ }^{23}$

O ACOPLAMENTO DE SUZUKI-MIYAURA (1979)

O acoplamento de Suzuki-Miyaura foi o que veio mais tarde cronologicamente, mas foi certamente um dos mais impactantes acoplamentos promovidos por paládio ${ }^{24}$. O primeiro relato desse acoplamento aconteceu somente em $1979^{3}$. A grande vantagem do acoplamento de Suzuki advém do emprego de alquilboranos ou boronatos, facilmente obtidos em grande variedade estrutural, em condições moderadamente básicas. Isto se deve à ativação do 
alquilborano ou boronato para que a transmetalação proceda com velocidades razoáveis. O mecanismo do acoplamento de Suzuki apresenta grandes semelhanças mecanísticas em relacão ao "acoplamento de Stille", diferindo no processo de ativação dos alquilboranos ou boronatos (Esquema 17).

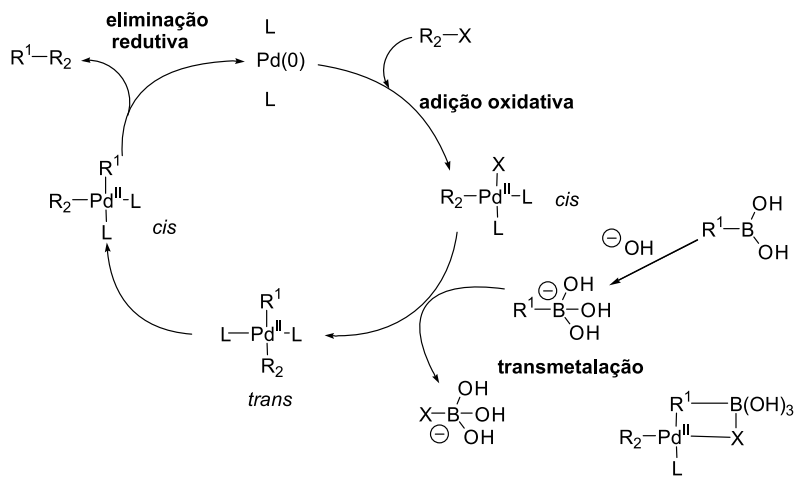

Esquema 17, O ciclo catalítico para o acoplamento cruzado de Suzuki ${ }^{24 a}$

O aspecto ainda um tanto polêmico no mecanismo proposto é a etapa de transmetalação, ainda pouco conhecida. No entanto, sabe-se que a presença da base é muitas vezes fundamental para o sucesso do acoplamento e que o passo determinante da velocidade de reação é a etapa de transmetalação. Uma possibilidade mecanística muito interessante envolve uma troca do halogênio no paládio por um grupo ${ }^{-} \mathrm{OR}$ (acetato, hidróxido, fosfonato, etc) (Esquema 18).

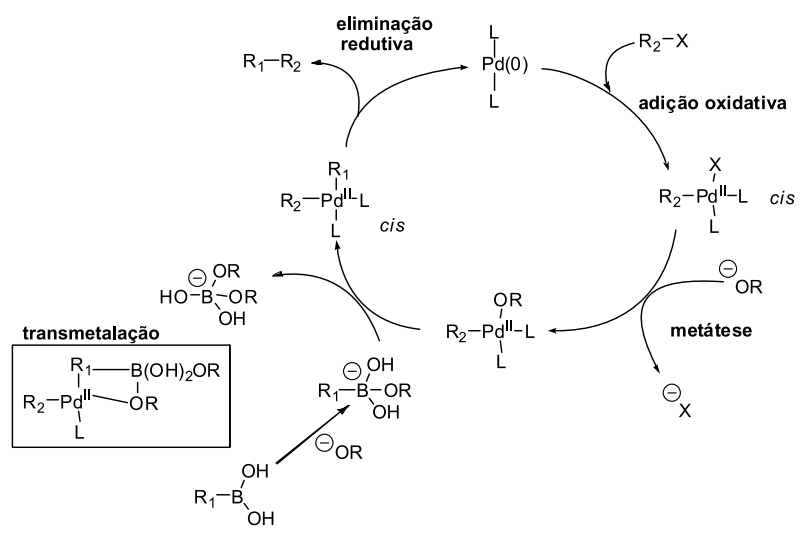

Esquema 18, Ciclo alternativo para o acoplamento de Suzuki ${ }^{24 a}$

A reação de Suzuki-Miyaura tem demonstrado um potencial sintético impressionante, sendo utilizada em escala de vários quilogramas na preparação de materiais para uso industrial e farmacêutico.

Um exemplo do uso eficiente desse acoplamento de Suzuki foi feito por Johnson ${ }^{25}$, na preparação de bis-azaçúcares contendo uma ponte carbônica, o que, em princípio, poderia levar à formação de "clusters" dessas unidades, acarretando alterações na atividade farmacológica. A preparação do brometo vinílico quiral para o acoplamento foi feito utilizando-se microorganismos e o alquilborano foi construído a partir da reação de hidroboração de alcenos, uma reação clássica (Esquema 19).

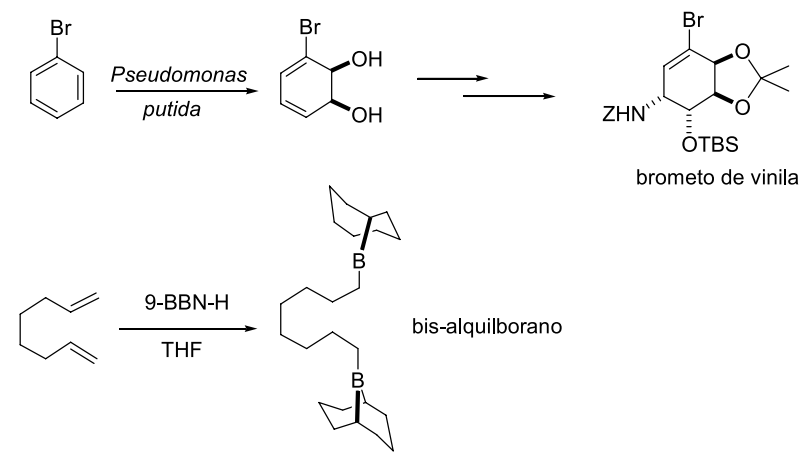

Esquema 19, Preparação do alquilborano para o acoplamento de Suzuki ${ }^{25}$

O curioso acoplamento de Suzuki duplo ocorreu de forma eficiente para fornecer sistemas razoavelmente complexos (Esquema 20).

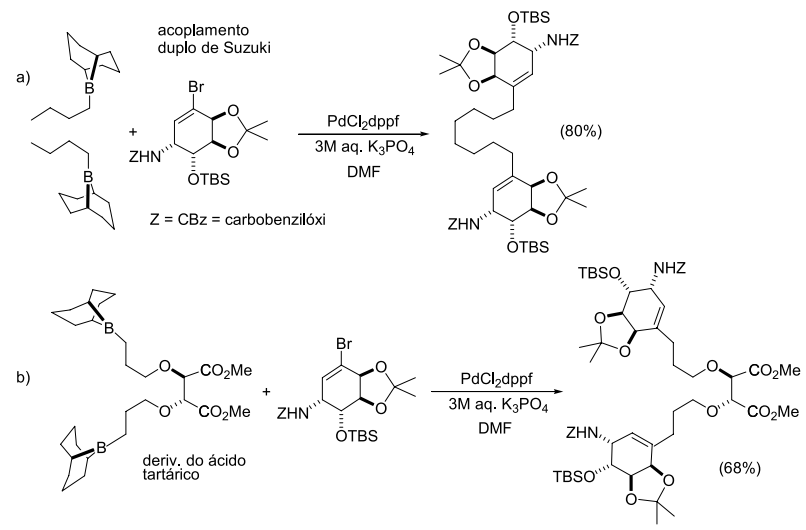

Esquema 20, O duplo acoplamento de Suzuki realizado por Johnson ${ }^{25}$

Uma vez realizado o acoplamento, os autores concluíram a preparação da unidade azaçúcar, ou 
piperidínica, envolvendo uma clivagem do anel cicloexênico com ozônio e ciclização da cetona formada com a amina primária liberada no meio reacional, após uma reação de hidrogenólise do grupo protetor do nitrogênio (carbobenzilóxi - Cbz).

Variantes do acoplamento de Suzuki têm aparecido com frequência na literatura. Uma das variantes mais eficientes da reação de Suzuki-Miyaura emprega ariltrifluoroboratos de potássio (Esquema 21) ${ }^{26}$.<smiles>Brc1cccc2ccccc12</smiles>
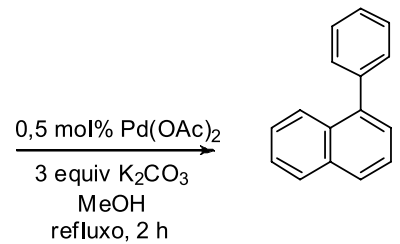

refluxo, $2 \mathrm{~h}$

Esquema 21. Exemplo do acoplamento de Suzuki empregando ariltrifluoroboratos de potássio ${ }^{26}$

Aplicações industriais ou em larga escala da reação de Suzuki-Miyaura são também frequentes na literatura. Por exemplo, a síntese do anti-MRS (anti-methicilline resistant Staphylococcus), realizada pelo grupo da Merck (Esquema 22) ${ }^{27}$.<smiles>CCOC(=O)C1=C([OH2+])C(C)C2N(C)C(=O)C12C(=O)O[Na]</smiles><smiles>NC(=O)CN1CCN(Cc2ccc3c(c2)C(=O)c2cc(B(O)O)ccc2-3)CC1</smiles>
${ }_{2}^{\ominus}$ OTf $\mathrm{Br}^{\ominus}$

1) $\mathrm{Pd}(\mathrm{dba})$, aq $\mathrm{Li}_{2} \mathrm{CO}_{3}$ $\mathrm{DMF} / \mathrm{CH}_{2} \mathrm{Cl}_{2}$ 2) $\mathrm{pH} 2,2-2,4, \mathrm{THF} / \mathrm{H}_{2} \mathrm{O}$

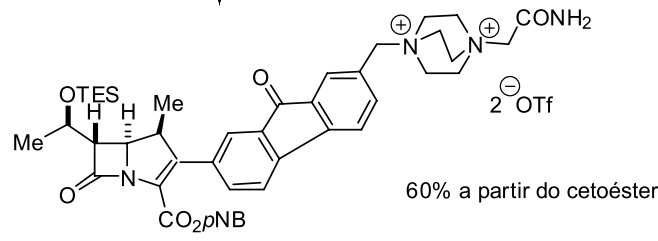

Esquema 22, Exemplo do acoplamento de Suzuki na preparação em larga escala de composto com atividade antibiótica ${ }^{27}$

\section{VISÃO GERAL DOS ACOPLAMENTOS CRUZADOS PROMOVIDOS POR PD}

Os acoplamentos cruzados e processos correlacionados, como por exemplo a reação de Heck, compartilham grandes semelhanças mecanísticas. Uma esquematização procurando integrar os processos conhecidos promovidos por paládio é apresentada no Esquema 23.

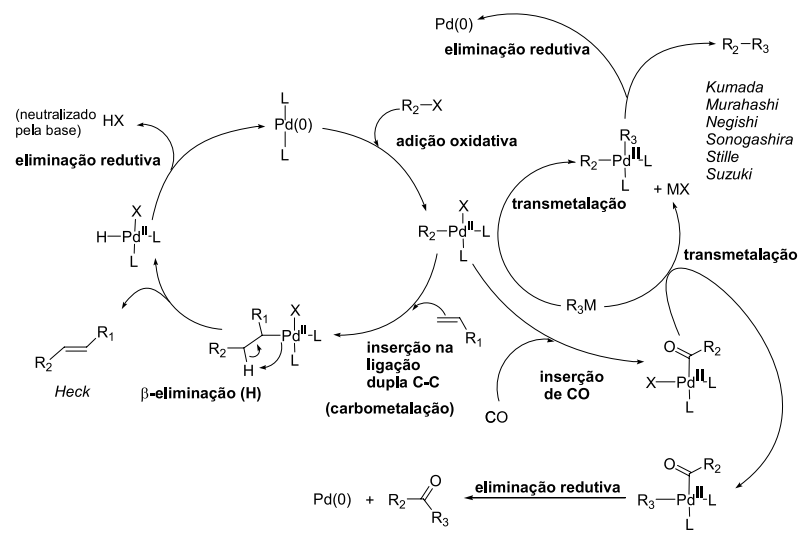

Esquema 23, Visão geral dos diversos acoplamentos cruzados, sua inter-relação com a reação de Heck e outras reações promovidas por Pd

\section{Conclusões}

Após 40 anos de estudos intensos e bem diversificados, as reações de acoplamento cruzado ganharam enorme projeção no cenário químico mundial, demonstrando serem processos extremamente eficientes e de grande versatilidade na formação de ligações $\mathrm{C}-\mathrm{C}$, um processo essencial na construção de moléculas orgânicas. Atualmente, são inúmeras as reações envolvendo os acoplamentos cruzados e suas variantes. Esses processos catalíticos são hoje empregados rotineiramente em laboratórios de pesquisas e laboratórios industriais envolvidos na produção de fármacos e novos materiais ao redor do mundo.

O Prêmio Nobel de Química de 2010 veio confirmar a grande importância desses processos químicos no cenário atual. A aplicação dessas reações de acoplamento cruzado tem permitido a síntese de novos materiais orgânicos, com maior eficiência, de forma controlada e menos agressiva ao ambiente. A oportunidade de contato direto com o Professor Akira Suzuki durante o BMOS14 foi um fator que provavelmente estimulará uma maior percepção dos químicos brasileiros quanto à importância e ao uso das reações de acoplamento cruzado.

\section{Referências}

1. Suzuki, A.; Angew. Chem. Int. Ed. 2011, 50, 6722. 
2. Negishi, E-I.; Angew. Chem. Int. Ed. 2011, 50, 6738.

3. Miyaura, N.; Yamada, K.; Suzuki, A.; Tetrahedron Lett. 1979, $20,3437$.

4. Para uma revisão geral dessas reações, consulte: (a) Metalcatalyzed Cross-coupling Reactions (Eds.: F. Diederich, P. J. Stang), Wiley-VCH, Weinheim, 1998; (b) Metal-Catalyzed CrossCoupling Reactions (Eds.: A. de Meijere, F. Diederich), $2^{\text {nd }}$ ed., Wiley-VCH, Weinheim, 2004.

5. Tamao, K.; Sumitani, K.; Kumada, M.; J. Am. Chem. Soc. 1972, 94, 4374.

6. Corriu, R. J. P.; Masse, J. P.; J. Chem. Soc. Chem. Commun. 1972, 144.

7. Uchino, M.; Yamamoto, A.; Ikeda, S.; J. Organomet. Chem. 1970, 24, C63.

8. Livro de resumos "Post OMCOS-XI Symposium: Thirty Years of the Cross-Coupling Reaction”, pag. 2-3, 27-29 de julho de 2001, Kyoto Research Park, Kioto, Japão.

9. (a) Karasch, M. S.; J. Am. Chem. Soc. 1941, 63, 2316; (b) Tamura, M.; Kochi, J. K.; J. Am. Chem. Soc. 1971, 93, 1483; (c) Tamura, M.; Kochi, J. K.; J. Organomet. Chem. 1971, 31, 289; (d) Tamura, M.; Kochi, J. K.; Synthesis 1971, 303.

10. Yamamura, M.; Moritami, I.; Murahashi, S.-I.; J. Organomet. Chem. 1975, 91, С39.

11. Murahashi, S.-I.; Yamamura, M.; Yanagisawa, K.-I.; Mita, N.; Kondo, K.; J. Org. Chem. 1979, 44, 2408.

12. (a) Negishi, E.-I.; Baba, S.; J. Chem. Soc. Chem. Comm. 1976, 596; (b) Baba, S.; Negishi, E.-I.; J. Am. Chem. Soc. 1976, 98, 6729; (c) Negishi, E.-I.; King, A. O.; Okukado, N.; J. Org. Chem. 1977, 42, 1821; (d) Negishi, E.-I.; Acc. Chem. Res. 1982, 15, 340.

13. Sonogashira, K. Tohda, Y.; Hagihara, N.; Tetrahedron Lett. 1975, 4467.

14. (a) Dieck, H. A.; Heck, F. R.; J. Organomet. Chem 1975, 93, 259; (b) Cassar, L.; J. Organomet. Chem. 1975, 93, 253.

15. (a) Sonogashira, K.; Yatake, T.; Tohda, Y.; Takahashi, S.; Hagihara, N.; J. Chem. Soc. Chem. Commun. 1977, 291; (b) Stephens, R. D.; Castro, C. E.; J. Org. Chem. 1963, 28, 3313. (c) Revisão do acoplamento de Sonogashira: Sonogashira, K. “Comprehensive Organic Synthesis”, Trost, B. M. Ed.; Pergamon Press, 1991, vol. 3; Coupling Reactions Between sp2 Carbon and sp Carbon Centers; pp 521.
16. Kosugi, M.; Kurino, K.; Takayama, K.; Migita, T.; J. Organomet. Chem. 1973, 56, C11.

17. Veja referência 8, págs. 18 e 19.

18. Grignon, J.; Pereyre, M.; J. Organomet. Chem. 1973, 61, C33.

19. (a) Kosugi, M.; Shimizu, Y.; Migita, T.; J. Organomet. Chem. 1977, 129, C36. (b) Kosugi, M.; Shimizu, Y.; Migita, T.; Chem. Lett. 1977, 1423; (c) Kosugi, M.; Sasazawa, K.; Shimizu, K.; Migita, T.; Chem. Lett. 1977, 301.

20. Milstein, D.; Stille, J. K.; J. Am. Chem. Soc. 1978, 100, 3636.

21. Para algumas revisões com dados de valor histórico, consulte: (a) Farina, V.; Krishnamurthy, V.; Scott, W. J.; Org. React. 1997, 50, 1; (b) Betzer, D. A.; Synthesis 1998, 522; (c) Ritter, K.; Synthesis 1993, 735; (d) Mitchell, T. N.; Synthesis 1992, 803; (e) Stille, J. K.; Angew. Chem. Int. Ed. 1986, 25, 508.

22. Casado, A. L.; Espinet, P.; J. Am. Chem. Soc. 1998, 120, 8978.

23. Smith, A. B.; Ott, G. R.; J. Am. Chem. Soc. 1996, 118, 13095.

24. Revisões: (a) Miyaura, N.; Suzuki, A.; Chem. Rev. 1995, 95, 2457. (b) Suzuki, A.; J. Organomet. Chem. 1999, 567, 147. (c) Suzuki, A.; In Metal-Catalyzed Cross-Coupling Reactions; Diederich, F., Stang, P. J. Eds.; VCH: Weinheim, Germany, 1998, pp 49-97. (d) Miyaura, N.; J. Organomet. Chem. 2002, 653, 54. (e) Suzuki, A.; J. Organomet. Chem. 2002, 653, 83.

25. Johns, B. A.; Johnson, C. R.; Tetrahedron Lett. 1998, 39, 749.

26. Molander, G. A.; Biolatto, B.; Org. Lett. 2002, 4, 1867.

27. Yasuda, N. J.; J. Organomet. Chem. 2002, 253, 279.

\section{Carlos R. D. Correia}

Instituto de Química, Unicamp, CEP 13083-970, Campinas, São Paulo, Brasil

*e-mail: roque@iqm.unicamp.br 